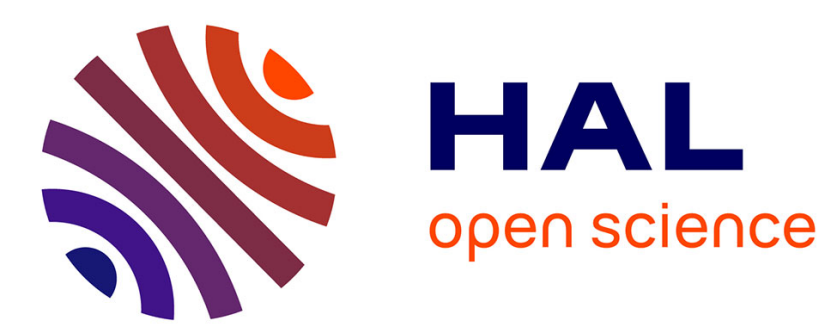

\title{
Neonatal Events Recognition using LBP descriptor and Wavelet Thresholding Technique
}

\author{
Adnan Al Alwani, Youssef Chahir
}

\section{To cite this version:}

Adnan Al Alwani, Youssef Chahir. Neonatal Events Recognition using LBP descriptor and Wavelet Thresholding Technique. 2014 International Conference on Multimedia Computing and Systems (ICMCS 2014), Jun 2014, Marrakech,, Morocco. pp.427 - 432, 10.1109/ICMCS.2014.6911171 . hal01168444

\section{HAL Id: hal-01168444 \\ https://hal.science/hal-01168444}

Submitted on 18 Sep 2018

HAL is a multi-disciplinary open access archive for the deposit and dissemination of scientific research documents, whether they are published or not. The documents may come from teaching and research institutions in France or abroad, or from public or private research centers.
L'archive ouverte pluridisciplinaire HAL, est destinée au dépôt et à la diffusion de documents scientifiques de niveau recherche, publiés ou non, émanant des établissements d'enseignement et de recherche français ou étrangers, des laboratoires publics ou privés. 


\section{Neonatal Events Recognition using LBP descriptor and Wavelet Thresholding Technique}

\author{
*Adnan AL ALWANI \\ GREYC-CNRS (UMR 6072), \\ University of Caen Lower Normandy \\ Caen, France \\ adnan.alalwani@unicaen.fr
}

\author{
Youssef CHAHIR \\ GREYC-CNRS (UMR 6072), \\ University of Caen Lower Normandy \\ Caen, France \\ youssef.chahir@unicaen.fr
}

\begin{abstract}
- this paper presents an approach for preterm events recognition using local Binary pattern LBP and wavelet thresholding. Unlike conventional approaches which adopt contact measurement of vital signal and develop models for monitoring the preterm in neonatal intensive care unit (NICU), our approach describes non-contact monitoring vital signal based on thermal video as a new premature pain infant profile (PPIP). An interest clinical vital signal is face temperature of neonate. In this study, we collaborate with premature Infant events 'pain and normal states' detection and recognition during daily care monitoring. The proposed approach is composed of a thermal video analysis component and an activity recognition component. A video analysis component contains features descriptor using histogram of Local Binary Pattern LBP in the first experiment, and applying wavelet thresholding technique on approximation wave coefficients of histogram feature in the second experiment. Activity recognition contains classification of premature events using classifiers. We present recognition results by considering a variety of classifiers.
\end{abstract}

Keywords- Local Binary pattern (LBP), wavelet thresholding, distance metric, preterm events recognition

\section{INTRODUCTION}

Vital signals are physical quantities measured from body and can be used to determine the physiological behaviors and statues. Monitoring of vital parameters is an important factor in neonatal intensive care unit. Examples of this signal include heart rate, breathing rate, body temperature and blood pressure. Vital signs are used in neonatal intensive care to help diagnose the condition of a baby in a critical state. The state of condition monitoring of an infant cannot be observed directly, but different states of health are associated with particular patterns of measurements [23]. Given observations of the respiration rate, blood sampling, and body temperature and so on, reasoning can therefore be made about the operation of the underlying physiological systems - e.g. whether they are functioning normally, or whether there seems to be evidence of some pathology. Most of vital signs measurements are taken by using contact probes. The measurements received by the cotside PC are affected not only by the physiological condition of the infant, but also by the state of the recording equipment. Measurements are frequently corrupted with occasional patterns, caused by particular clinical procedures or changes to probes or equipment. The most obvious cause of occasional readings is a probe disconnection. When probes become physically disconnected from the baby, readings will tend to fall to zero. Further details on physiological monitoring procedures in the NICU are available, for example, in [1].

Relevant previous work on monitoring in intensive care, applied to the types of patterns based on contact probes are broadly categorized into three fields. Work based on statistical time series analysis was explored by some inserters. A useful reviews of some of the applications of statistical time series analysis to intensive care monitoring is given by [2] [3] and [4]. By contrast, another body of work is based on using domain knowledge to formulate high-level representations of particular patterns or situations, then to find suitable abstractions of the data in order to apply some matching rules. In this type of work, the goal is to describe what is happening. A more extreme example of the idea of abstracting data and the descriptions of clinical experts is given by [5]. At the most specific level, there has been work on identification of particular individual patterns in monitoring data, for example to diagnose infection or particular respiratory problems. An example of this work is reported by [6].

In general, thermal video analysis has significant challenges, it excite some researchers interesting to develop a relevant techniques for example human activity detection and recognition using standard dataset. Attempts were explored by researchers to develop approaches to detect and recognize human activities from thermal video can be found in [10-14]. The implementation details of these approaches are beyond the scope of this paper.

Medical thermal imaging (also known as Digital Infrared Thermal Imaging DITI or Medical Infrared Imaging) uses ultra-sensitive thermal imaging camera to produce highresolution diagnostic images of the body. These images can be used to detect areas of temperature changes associated with areas of high metabolic activity, inflammation and enhanced circulation. This makes the images useful in diagnosing pain and injury, circulatory pathology and in 
monitoring treatment. Recently, thermal imaging was introduced as non-contact probe in neonatal intensive care for condition monitoring. For instance, thermal video can be used to maintain thermal signature of baby's skin, as well as thermal video analysis depend on temperature signature of object under study. Moreover, Dynamic shape of recorded temperature specifies the texture characteristics of thermal image and it is used to detect meaningful behavior of the target.

As mentioned, to overcome the limitation of using the contact probes in NICU, thermal video works more efficiently by deriving and using information of local thermal signature from infants' body. However, this kind of imaging present body configuration in form of thermal pixels, also, it has remote accesses facility, and may be used as visual alarm in condition monitoring. There are attempts in neonatal condition monitoring based non-contact measurements, including neonatal respiratory monitoring based on thermal video [17], and Contact-free measurements of cardiac pulse based on the analysis of thermal imagery [18]

Recently detection and recognition physiological events in neonatal based thermal video have been little explored. In this work, we report a method of preterm physical responses detection and recognition. The motivation in this work is to explore the power of Local Binary Pattern and wavelet thresholding technique as local thermal descriptors for neonates' events detection. We have made an improvement on the features description by adopting pairwise distance between feature vectors. For instance, Hausdorffs distance function and Entropy distance functions are introduced to improve the features representation. To evaluate our method we employ a support vector Machine SVM, and K nearest neighbor, classifiers. Moreover, we recognize neonates' pain response using variety of classifiers. The purpose of using multiple classifiers is to provide a comparison between the feature descriptions, because there were no experiments and methods were focused on the neonatal events recognition based on thermal imaging using NICU dataset environment. Experiments show that the recognition task achieves reasonable results on NICU dataset based thermal video.

The paper is organized as follows. Section 2 presents the outline of the method, section 3 describes problem formulation, where the proposed method is elaborated from features descriptions to evens recognition by a variety of classifiers. We proceed to sections 4 and 5 to explain experimental setups and results respectively. Section 6 concludes the paper.

\section{METHODOLOHY}

Our approach is composed of two stages to obtain the local description of the infant's events. The first stage is to describe local thermal features by using LBP and then concatenate these features into one dimensional histogram vector. To make the histogram more efficient, we have evaluated the similarity matrix between histogram features vectors using Hausdorff's and entropy distance function, respectively. In the second stage, a feature descriptor is obtained by producing an approximation coefficients threshold of histogram vectors which are computed using wavelet transform WT. As in the first stage, similarity matrices are adopted for improving the feature descriptor. Fig. 1 shows the basic steps of our method. The first step defines ROI area around face of object. Then local patch of size $k^{*} k^{* t}$ is defined. LBP is computed in second step. And the classification task is performed in the last steps.

\section{PROBLEM FORMULATION}

In this work, local feature descriptions using LBP and subsequent contributions are described. The feature description from LBP is subject to some variation. With the use of wavelet threshold technique, we can improve the feature description which leads better recognition accuracy.

\section{A. Local Binary Pattern Descriptor}

LBP has originally been proposed for texture analysis and classification [9]. Recently, it has been applied on face recognition [7][8], and facial expression recognition [19].

We denote a video sequence as $\mathrm{S}=\left\{\mathrm{I}_{1}, \mathrm{I}_{2}, \ldots, \mathrm{I}_{\mathrm{n}}\right\}$, where $\mathrm{I}_{\mathrm{k}}$ is a $\mathrm{r} \times \mathrm{c}$ image frame. In contrast, treating the video as a spatio-temporal data volume and for entire spatio-temporal location we denote by $\mathrm{W}(\mathrm{v})$ the local patch volume $\mathrm{v}$ of ROI. Furthermore, denote by LBP $(\mathrm{W}(\mathrm{v}))$, or $\mathrm{M}(\mathrm{v})$ the LBP of local patch. Hence, using the $2 \mathrm{D}$ patch to slide over the video sequence and get the local features. The value of LBP code of a pixel $\left(\mathrm{x}_{\mathrm{c}}, \mathrm{y}_{\mathrm{c}}\right)$ within the patch is obtained as in

$$
L B P=\sum 0 P-I I\left(g_{p}-g_{c}\right) 2^{P}, x=\left\{0_{0 \text { otherwise }} \text { if } x>=t\right.
$$

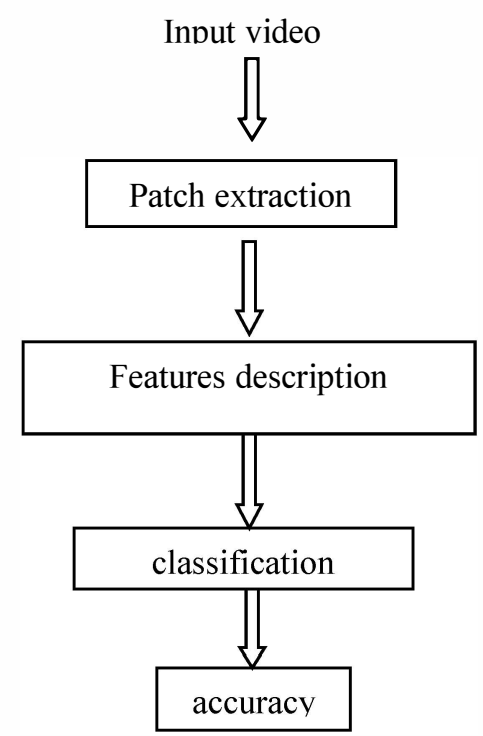

Figure 1. method of events-based feature description and recognition. 


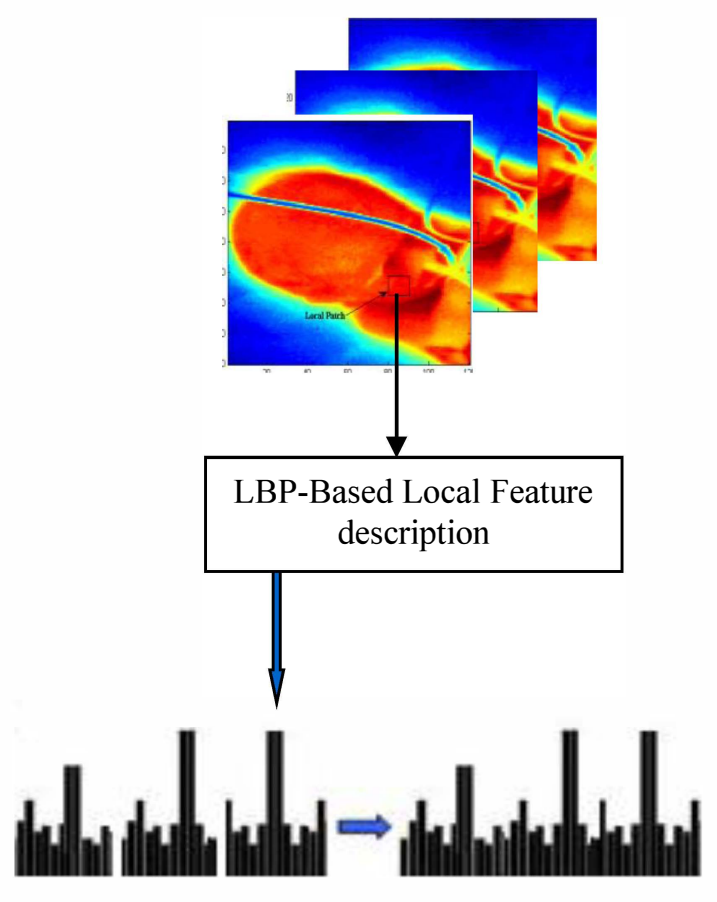

Figure 2. Histogram Features extraction from Local patch

After the LBP labeled image $\mathrm{fl}(\mathrm{x}, \mathrm{y})$ has been obtained for all patch pixels, the LBP histogram can be defined as in

$$
H_{i}=\sum_{x, y} h\{f l(x, y)=i\} i=0, \ldots . n-1,
$$

Where, $n$ is the number of different labels produced by the LBP operator. Having constructed the histogram feature of local patch, histograms from these planes are concatenated into a single histogram vector $\mathrm{H}=\{\mathrm{H} 1, \mathrm{H} 2, \ldots, \ldots \mathrm{Hn}\}$. Fig. 2 shows example of extracting histogram features vector from local patch of video sequence and denote this LBPH. Figure 3 illustrates example result of LBPH extracted from subject example showed in figure 2 .

\section{B. Pair wise distance representation}

The objective of this section is to utilize the remarkable discriminative ability of the similarity based representation. More specifically, we have improved the above descriptor by using pairwise distance function. In order to accomplish that, the histogram features is transformed into similarity space. Moreover, as each video is represented by a LBPH, the distance function is used to compute the similarity value between two sets histogram vectors. Several distance metric methods have been developed to compute the distance between two sets of vectors [21].

Here, a task where new events are discriminated on the rule of pairwise recognition to events of a training set is adopted. The general mathematic formulation for similarity space is illustrated as follows :
We denote by $d\left(h_{i}, h_{j}\right)$ the distance between two histogram vectors, the similarity matrix is defined as in

$$
D i, j)=\sum_{i, j=1 \ldots N} d\left(h_{i}, h_{j}, Q\right)
$$

Where, Q specifies the distance function used to compute the similarity matrix. Furthermore, let $\mathrm{X}$ and $\mathrm{Y}$ be two nonempty subsets of a metric space. We define their Hausdorff distance as in.

$$
d_{H}(X, Y)=\max \left\{\sup _{x c X} \inf _{y c Y} d(x, y), \sup _{y c Y} \inf _{x c X} d(x, y)\right\} .
$$

Where sup represents the supremum and inf the infimum similarly, Entropy function is also introduced to evaluate the discriminative power and efficiency for feature description improvement. The entropy distance between two random variables can be defined to be the difference between their joint entropy and their mutual information as

$$
D_{I}(X, Y)=H(X, Y)-H(X ; Y)
$$

Where, $\mathrm{H}(\mathrm{X}, \mathrm{Y})=\sum \mathrm{p}(\mathrm{x}, \mathrm{y}) \log \mathrm{l} / \mathrm{p}(\mathrm{x}, \mathrm{y})$, is the joint entropy of X,Y.

For the final step of the feature extraction, linear Discriminant analysis performed on the pairwise feature set. Basically, LDA is based on the class specific information which maximize the ratio of between-class scatter and the within-class scatter matrix. the LDA algorithm looks for the vectors in the embedding space to create the between classes discrimination. Thus, utilizing LDA on the pairwise features, robust feature space can be obtained that discriminate the set vectors of each class.

\section{Wavelet Thresholding of Histogram Features}

Feature description is the most important information for preterm events recognition; it is used to distinguish one kind of physiological event different from another kind of event. As described in the last section, the LBP approach to event recognition involves extracting histogram feature vector from images sequences. We believe that wavelets may be a better choice to improve feature description so we propose to use wavelet multi-resolution analysis [22], and wavelet thresholding technique [16]. More details of wavelet signal analysis can be found in [20].

Our goal is to apply wavelet thresholding or wavelet shrinkage technique on extracted approximation coefficients of histogram vector, the resulting feature define the feature description based wavelet threshold we call this LBPWT. In our implementation, multi-levels wavelet is used to encodes histogram features vector $H=\left\{h_{1}, \ldots . h_{n}\right\}$ as approximation $\Omega$ components, and the result is vector containing an approximation wave coefficients $\phi(\Omega)$. It is observed that features of the approximation components change smoothly, which is a suitable property for discrimination. The approximation components are also known to be a robust and distortion tolerant feature space for many pattern recognition applications. 

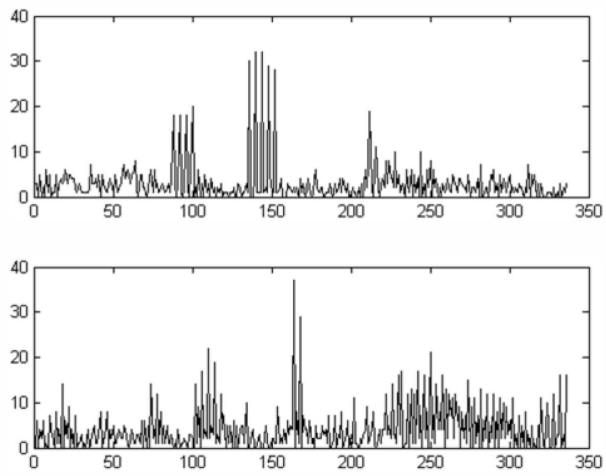

Figure 3. Concatenated Histogram vector, (top) pain response,(bottom) normal response.

The histogram vector is decomposed to a certain level and the coefficients at the lowest level are selected to form feature description vector. One Dimensional J - level wavelet decomposition on a vector $\mathrm{H}=\{$ represents the coefficients by a set of approximation and details components $\left[a_{\mathrm{J}},\left\{\mathrm{d}_{1}, \mathrm{~d}_{2}, \ldots . . \mathrm{d}_{\mathrm{j}}\right\}_{\mathrm{j}=1 . . \mathrm{J}}\right]$. Where $\left.\mathrm{h}_{1}, \ldots . \mathrm{h}_{\mathrm{n}}\right\} \mathrm{a}_{\mathrm{J}}$ is a low resolution approximation of the original signal, and $d_{j}$ are detail coefficients containing the details of the signal at different scales. Figure 4 shows an example result of wavelet decomposition at two levels, where a wave coefficients and details of the histogram are showed. Note that, for this two levels decomposition all wave coefficients are illustrated in this figure.

One of the advantages of using wavelet thresholding is its potential to deal with noise. wavelet thresholding characterized as decomposition of the signal or into wavelet coefficient with a given threshold value, and shrinking these coefficients close to zero to reduce the effect of noise in the data, then applying thresholding technique to find smoothing version of wavelet components. There exist various methods for wavelet thresholding developed by [15],[16],[24], which rely on the choice of a threshold value. In this experiment, we adopt hard thresholding technique as a threshold function defined as.

$$
T=\left\{\begin{array}{l}
1, \quad|x|>t \\
0 \text { otherwise }
\end{array}\right.
$$

Where $\mathrm{t}$ is the threshold value and can be calculated using the formula below

$$
t=\sigma \sqrt{ } 2 \log (n)
$$

where, $\mathrm{n}$ being the signal length, $\sigma$ being signal variance which can be estimated from wavelet coefficients. As a result, the extracted approximation coefficients from wavelet threshold are encoded as a features descriptor. Fig. 5 shows the plot of the thresholding wave coefficients.
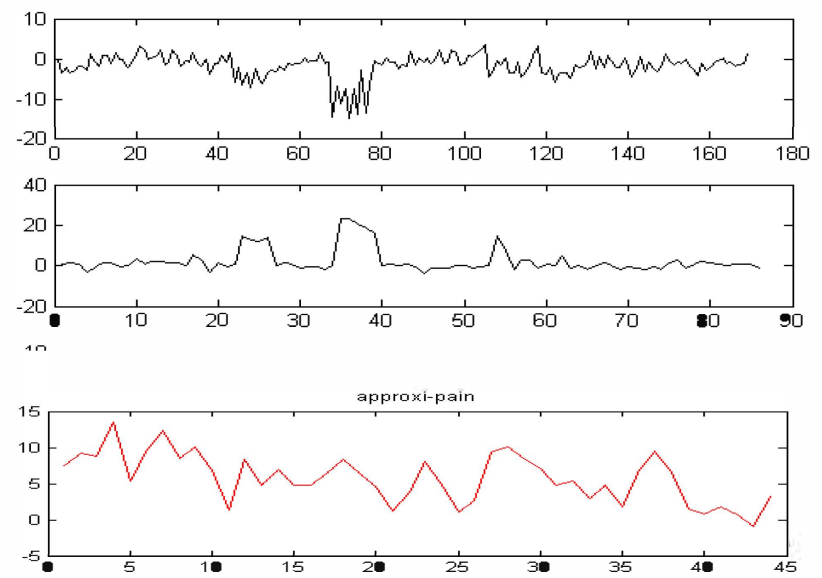

Figure 4. Wave coefficients of illness state (top panel) detiales dl and d2 components, (nottom panel) apprixination component.
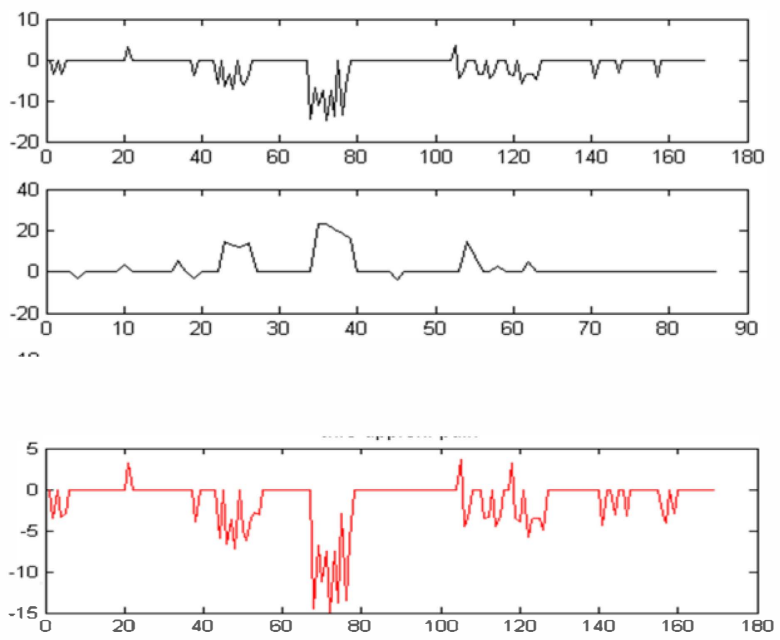

Figure 5. Wave coefficients results after thresholding for 2 level decomposition, (top panel) detailes $\mathrm{d} 1$ and $\mathrm{d} 2$, (bottom panel) an approximation coefficients

\section{EXPERIMENTAL RESULTS}

For events recognition experiments, we chose to use the dataset of neonatal in NIUC system based thermal video. The dataset are provided by preterm ANR project. These dataset contains several thermal videos monitoring of the Premature Infants, we encode among of these videos a 20 clips which represent an event of premature pain state, as well as a 20 clips represent a normal state event of premature. Moreover each event was collected from 20 different infants. Altogether, the dataset contains 40 samples of two events class problem. The length of each sample is equal to 20 frames.

A patch of size $11 \times 11$ pixels is defined over infant's face as local interest area. The features vectors and corresponding distances matrices were described in sections 2.2 and 2.3. 
For LBP the $\mathrm{P}$ parameter is set to $\mathrm{P}=4$. Doubechies wavelet function is used with 3 decomposition levels.

We start the experiments with the recognition task based on traditional LBPH descriptor. Thus, to compare the performance enter the classifiers, pairwise distance based experiments were designed in this connection. For further experiments, features descriptor was encoded as a wavelet threshold-based technique. Conventional classifiers of SVM, and k-NN, are used for recognition task.

\section{A. Results}

To evaluate the proposed method, we conduct the recognition results on the dataset described above. In each of our approaches experiment, the training process is done on a 20 samples (neonates), and testing phase was performed on another 20 samples.

Because no available of such methods that are focused on the neonate physiological events recognition, we did not attempt a comparison with other methods. Rather, we provide results only to show that our approach works well on a diverse range of data such as neonates' events recognition based thermal video.

We first applied the LBPH directly with classifiers. The recognition rates of two classifiers are listed in Table1. Since LBP is considered to be good texture descriptor of face, it seems poor operator as shown in table I. Furthermore, we continued our experiments to the wavelet threshold-based LBP descriptor, and we call this WTLBP. Moreover, WTLBP applied directly with recognizers and the recognition rate is summarized in Table II. Since LDA is a good tool to separate the underlying feature space, therefore, a better feature space is created by applying LDA classification over the pairwise features. Hence, LDA-based experiments over pairwise features are listed in table III, showing both the Hausdorffs distance function and Entropy function experiment based methods. The experiments results show that Hausdorff distance outperforms over the Entropy distance and LBP over WTLBP. In summary, by combining the LBPH and pairwise distance, we can obtain better features to represent thermal signature in thermal sequence.

As a comparison, the performance of LBPH descriptor in combination with pairwise distance is better in events recognition of premature from thermal video.

\section{CONCLUSION}

In this paper, we have applied LBP as a descriptor of local-based approach for neonatal activity recognition from thermal video, and shown LBP to be suitable for the description of thermal video containing information about neonates events. We have extended feature descriptor by considering the pairwise distance between histogram features vectors. We showed how the use of pairwise distance based LBP descriptor gave rise to an efficient events descriptor. We further extend LBP descriptor by encounter Wavelet thresholding technique. Furthermore, we applied wavelet Thresholding on LBP histogram by compute the threshold value from wavelet coefficients. The experimental results
TABLE I. RECOGNITION RATE (\%) OF DIRECT LBPH

\begin{tabular}{|c|c|c|}
\hline Method & K-NN & SVM \\
\hline LBPH & 80 & 65 \\
\hline
\end{tabular}

TABLE II. RECONITION RATE (\%) OF DIRECT WT LBP

\begin{tabular}{|c|c|c|}
\hline Methods & k-NN & SVM \\
\hline WTLBP & 65 & 75 \\
\hline
\end{tabular}

TABLE III. RECONITION RATE (\%) OF APPLAYING LDA OVER PAIRWISE FEATURES VALUE

\begin{tabular}{|c|c|c|}
\hline methods & k-NN & SVM \\
\hline LBP+Hausdorf. & 75 & 70 \\
LBP+ Entropy & 80 & 85 \\
\hline WTLBP+Hausdorf. & 50 & 85 \\
WTLBP+ Entropy & 50 & 50 \\
\hline
\end{tabular}

reveal that LBP tend to be good candidate for events description and recognition. The best accuracy has been obtained by using the pairwise metrics between histogram vectors set.

\section{ACKNOWLEDGEMENTS}

The authors wish to thank ANR Preterm, for providing datasets of neonatal conditions monitoring of daily care.

The first author would like to thank his employer, university of AL Anbar for their continuous supporting during study time.

\section{REFERENCES}

[1] J.M. Rennie and N.R.C. Roberton, "A Manual of Neonatal Intensive Care," Hodder Arnold, 2001.

[2] R. Fried, U. Gather, and M. Imhoff, "Pattern Recognition in Intensive Care OnlineMonitoring," In Sonderforschungsbereich (SFB) 475, volume 1, pages 509-512. University of Dortmund, 2003.

[3] S. Charbonnier, G. Becq, and G. Biot, "On-Line Segmentation Algorithm for Continuously Monitored Data in Intensive Care Units", IEEE Transactions on Biomedical Engineering, 51(3):484-492, 2004

[4] S.W. Hoare and P.C.W. Beatty, "Automatic artifact identification in anaesthesia patient record keeping: a comparison of techniques," Medical Engineering and Physics, 22: 547-553, 2000

[5] S. Miksch, Horn, C Popow, and F. Paky, "Utilizing Temporal Data Abstraction for Data Validation and Therapy Planning for Artificially Ventilated Newborn Infants," Artificial Intelligence in Medicine, 8(6):543-576, 1996.

[6] M.P. Griffin and J.R. Moorman, "Toward the earlear diagnosis of neonatal sepsis and sepsis-like illness using novel heart rate analysis," Pediatrics, 107(1):97-104, 2001.

[7] T.,Ahonen, ,A. Hadid, M. Pietikäinen, "Face Description with Local Binary Patterns. Application to Face Recognition," IEEE Transactions on Pattern Analysis and Machine Intelligence 28(12). 2037-2041.2006. 
[8] T.,Ahonen, ,A. Hadid, and M. Pietikäinen, "Face recognition with local binary patterns," In: Pajdla, T., Matas, J.G. (eds.) ECCV 2004. LNCS, vol. 3021, pp. 469-481. Springer, Heidelberg, 2004.

[9] T. Ojala, M. Pietikäinen, and T. Mäenpää, "Multiresolution grayscale and rotation invariant texture classification with local binary patterns," IEEE Transactions on Pattern Analysis and Machine Intelligence, pp. 971-987,2022.

[10] M. Aren, and K. Jungling, "feature based person detection beyond the visible spectrum," IEEE CVPR WorkShops. 2009.

[11] M. Bertozzi, "Pedstrain detection in infrared images" Proc. IEEE, Intelligent Vehicles Symp. Columbus, OH.2003.

[12] J. Davis, and V. Sharma, "Robust background-subtraction for person detection in thermal imagery," IEEE, Int. Wkshp. On Object Tracking and Classification beyond the Visible Spectrum. 2004.

[13] A. Mark, K. James, and W. Davis, "A two-stage template approach to person detection in thermal imagery," Proceeding Workshop Applications of Computer Vision. 2005.

[14] W. Wang, J. Zhang, and C. Shen, "Improved detection and classification in Thermal images," ICIP.2010.

[15] L. David, and M. Iain, "Adapting to unknown smoothness via wavelet shrinkage," Journal of the American Statistical Association, 90(432), pp.1200-1224.1995.

[16] L. David, and M. Iain, "Ideal spatial adaption via wavelet shrinkage, ” Biometrika. vol. 81,pp 425-455.1994.

[17] M. Garbey, N. Sun, A. Merla, and I. Pavlidi, "Contact-free measurements of cardiac pulse based on the analysis of thermal imagery," Technical Report Number UH-CS-04-08.

[18] A. Abbas K.,K. Heimann, K. jergus, T. Orlikowsky, and S. leonhardt, "Neonatal non-contact respiratory monitoring based on real time infrared thermography" Biomedical Engineering onLine. 2011.

[19] C. Shan, "Facial Expression recognition based on Local Binary Patterns. A comprehensive study," Image and Vision Computing.2008.

[20] Stephane G mallat, "A wavelet tour of signal processing," Elsevier Inc,3rd Edition. 2008.

[21] Victor Bryant, " Metric Spaces: Iteration and Application," Cambridge University Press.1985.

[22] I. Doubechies, "Orthonormal Bases of Compactly Supported Wavelets," Comm. Pure Applied Math, Vol. 41, 1988.

[23] John A. Quinn, "Bayesian Condition Monitoring in Neonatal Intensive Care," phD. Dissertation, University of Edinburgh 2007.

[24] Pierre Moulin,1994, "Wavelet thresholding techniques for power spectrum estimation," IEEE Transactions on Signal Processing. 42:3126-3136.1994.

[25] N. Cristianini and J. Taylor, "An introduction to support vector machines and other kernel-based learning methods", Cambridge UP, 2000 . 